\title{
Boundary Expansions for Spline Interpolation
}

\author{
By W. D. Hoskins
}

\begin{abstract}
An explicit method is given for deriving the formulae for derivatives of the spline of order $m+1$ at two boundaries $x=a, x=b$ in terms of known function values and computed $m$ th derivatives of the spline.
\end{abstract}

1. Introduction. General formulae for computing the spline $s(x)$ of degree $m+1$ on a partition $a=x_{0}<x_{1}<\cdots<x_{n}=b$ of the interval $[a, b]$ have been considered with various types of boundary conditions in several previous papers (Ahlberg, Nilson and Walsh [1], Späth [5]).

However, the spline interpolation problem may always be formulated as the solution of a matrix equation where the matrix of coefficients is of band type with width $m+1$. The special case of periodic boundary conditions yields a predominantly band matrix which is of circulant form (Hoskins and King [3]).

In the case when $s^{(a)}\left(x_{i}\right)(j=0, n ; q=1,2, \cdots, m-1)$ or, more generally, when linear combinations of these quantities are given, or, alternatively, when they merely require to be determined from the computed set of $m$ th derivatives, it is not apparent from the development by Ahlberg et al. [1, pp. 124-132] that they may be obtained explicitly for all forms of boundary conditions solely in terms of values of the function and $m$ th derivatives. Further, the explicit form for these conditions is such that the band structure of the matrix of coefficients may be retained.

2. Development of the Expansions. The polynomial spline $s(x)$ of degree $m+1$ has as its $(m+1)$ st derivative

$$
s^{(m+1)}(x)=\frac{s_{i}^{(m)}-s_{i-1}^{(m)}}{h_{j}}, \quad j=1,2, \cdots, n,
$$

where $x_{i-1} \leqq x \leqq x_{i}, h_{i}=x_{i}-x_{i-1}$ and $s_{i}^{(m)}$ denotes the $m$ th derivative of $s(x)$ evaluated at $x=x_{i}$. Now the function $s(x)$ can be represented over the complete range $\left[x_{0}, x_{n}\right]$ in the form

$$
s(x)=\sum_{k=0}^{m} \frac{x^{k}}{k !} s_{0}^{(k)}+\sum_{t=0}^{n-1} \frac{d_{t}}{(m+1) !}\left(x-x_{t}\right)_{+}^{m+1}
$$

where

$$
\begin{aligned}
z_{+} & =z, \quad z \geqq 0, \\
& =0, \quad z<0
\end{aligned}
$$

(Powell [4]). Using Eqs. (2.1) and (2.2) it is seen that

Received December 9, 1970, revised December 6, 1972.

AMS (MOS) subject classifications (1970). Primary 41 A15.

Key words and phrases. Multipoint expansions, spline interpolation. 


$$
\begin{aligned}
& d_{0}=\left(s_{1}^{(m)}-s_{0}^{(m)}\right) / h_{1}, \quad \text { and } \\
& d_{t}=\left(s_{t+1}^{(m)}-s_{t}^{(m)}\right) / h_{t+1}-\left(s_{t}^{(m)}-s_{t-1}^{(m)}\right) / h_{t}, \quad 0<t<n-1,
\end{aligned}
$$

and, if Eq. (2.2) is expanded about the values $x_{k}(k=1,2, \cdots, m-1)$, then

$$
s_{k}=\sum_{i=0}^{m} \frac{x_{k}^{i}}{i !} s_{0}^{(i)}+\sum_{i=0}^{k-1} \frac{d_{i}}{(m+1) !}\left(x_{k}-x_{i}\right)^{m+1}
$$

with slight rearrangement producing

$$
\begin{aligned}
\sum_{i=1}^{m-1} \frac{x_{k}^{i}}{i !} s_{0}^{(i)}=s_{k}-s_{0}-\sum_{i=0}^{k-1} \frac{\left(x_{k}-x_{i}\right)^{m+1}}{(m+1) !} \cdot d_{i}-\frac{x_{k}^{m}}{m !} s_{0}^{(m)} & \\
k & =1,2, \cdots, m-1 .
\end{aligned}
$$

The complete set of Eqs. (2.4) may be written in matrix form as

$$
A \mathrm{v}=\mathrm{b}
$$

where $A=\left\{x_{i}^{j}\right\}$ and is $(m-1) \times(m-1), \mathrm{v}=\left\{s_{0}^{\prime}, s_{0}^{\prime \prime} / 2 !, \cdots, s_{0}^{(m-1)} /(m-1) !\right\}^{T}$ and the vector $b$ has elements

$$
b_{k}=s_{k}-s_{0}-\sum_{i=0}^{k-1} \frac{\left(x_{k}-x_{i}\right)^{m+1}}{(m+1) !} \cdot d_{i}-\frac{x_{k}^{m}}{m !} s_{0}^{(m)}, \quad k=1,2,3, \cdots, m-1 .
$$

The quantities $d_{i}$ appearing on the right-hand side of Eq. (2.5) are of course already given from Eqs. (2.3) in terms of $m$ th derivatives, hence $\mathbf{v}$ defined as the solution of (2.5) gives the required expansions for $s_{0}^{(a)}(q=1,2, \cdots, m-1)$.

The coefficient matrix $A$ is a special form of the Vandermonde matrix and possesses a well-known inverse (Gregory and Karney [2]) so that explicit expansions for the elements of $\mathbf{v}$ could be obtained if desired. However, from the computing aspect it is more economical to work directly with the system of Eqs. (2.5) when numerical values for the elements of $\mathrm{v}$ are required. In particular, if the partition of $\left[x_{0}, x_{n}\right]$ is uniform and $x_{i}=x_{0}+i h$ where $h=\left(x_{n}-x_{0}\right) / n$ and $i=0,1,2, \cdots, n$, then Eq. (2.5) can be simplified by associating the appropriate powers of $h$ with the derivatives appearing in $\mathrm{v}$, and the matrix $A$ becomes $A=\left\{i^{i}\right\}$. In this case, the vector $\mathrm{v}$ is easily obtained by solving the equivalent set of equations

$$
U_{\mathbf{v}}=L \mathbf{b}
$$

where $U=\left\{\Delta^{i} 0^{i}\right\}$ and $L=\left\{(-1)^{i+i}\left(\begin{array}{c}i \\ i\end{array}\right)\right\}$, with $\Delta^{i} 0^{i}$ representing the $i$ th difference of $\left.i^{i}\right|_{i=0}$. Equation (2.6) is such that $U$ is an upper triangular matrix and thus the determination of $\mathbf{v}$ is easily effected.

Department of Computer Science

University of Manitoba

Winnipeg, Manitoba, Canada

1. J. H. Ahlberg, E. N. Nilson \& J. L. Walsh, The Theory of Splines and Their Applications, Academic Press, New York, 1967. MR 39 \#684.

2. R. T. GREgORY \& D. L. KARNEY, A Collection of Matrices for Testing Computational Algorithms, Wiley, New York, 1969. MR 40 \#6752.

3. W. D. Hoskins \& P. R. KING, "Interpolation using periodic splines of odd order with equi-spaced knots," Comput. J., v. 15, 1972, pp. 283-284. England.

4. M. J. D. Powell, On Best $L_{2}$ Spline Approximations, AERE Report TP264, Harwell,

5. H. SpÄTH, Die Numerische Berechnung von interpolierenden Spline-Funktionen mit Blockunterrelaxation, Universität (TH) Karlsruhe, Karlsruhe, 1969. MR 42 \#659. 